\title{
Orientações para o sequenciamento das instruções em um objeto de aprendizagem
}

\author{
Maria Lucia Pozzatti Flôres, Ulbra-SM, PGIE/UFRGS, mlflores@ terra.com.br \\ Liane Margarida Rockenbach Tarouco, Prof ${ }^{a}$.Dr ${ }^{a}$. PGIE/UFRGS, liane@ penta.ufrgs.br \\ Eliseo Berni Reategui, Prof.Dr. PGIE/UFRGS, eliseoreategui@gmail.com
}

\section{Resumo}

O sequenciamento das instruções em um objeto de aprendizagem (OA) influi diretamente na maneira como o estudante vê e assimila a informação, facilitando o domínio de novos conceitos e idéias, proporcionando ao aluno uma aprendizagem significativa. Atualmente existe pouca discussão em torno de uma metodologia genérica para o desenvolvimento do OA mostrando como construí-lo e dando suporte ao seu uso instrutivo. Neste artigo serão mostradas algumas orientações para o sequenciamento das instruções em um OA. Ainda, será demonstrada a utilização prática das teorias apresentadas no planejamento de quatro objetos de aprendizagem.

Palavras chave: Seqüência da instrução. Objeto de aprendizagem.

\section{Guidelines for the sequencing of instructions in a learning object}

\begin{abstract}
The sequencing of instructions on a learning object (OA) directly influence the way students perceive and assimilate information, facilitating the mastery of new concepts and ideas, thus providing students with a meaningful learning. Currently there is few discussions about a generic methodology for development of OA, showing how to build it, and how to provide support for its instructional use. This article will show some guidance for the sequencing of instructions in an OA. Also, it's demonstrated the practical use of the theories presented in the planning of four objects of learning.
\end{abstract}

Keywords: Instruction sequence. Learning object.

\section{Introdução}

Um tipo de material didático digital que está sendo muito usado para subsidiar a aprendizagem é o objeto de aprendizagem (OA). Wiley (2000) entende que os objetos de aprendizagem são elementos de um tipo novo de instrução por computador baseado no paradigma "orientado ao objeto" da informática. Eles são criados em módulos que podem ser reusados em diferentes contextos (Dahl \& Nygaard, 1966). Ainda, os OAs podem ser entidades digitais distribuídas pela Internet, o que significa que todos podem acessá-los e usá-los simultaneamente.

Entretanto, é preciso refletir como estruturar o OA para que ele tenha instruções organizadas, contextos ricos de experiência que possam promover uma aprendizagem significativa. Para Merril (1992), a aprendizagem é facilitada caso se apresente aos alunos "organizadores estruturantes e idéias ancoráveis", isto significa que as instruções devem ser organizadas a partir de eixos estruturados em torno de uma idéia chave, de um tema.

Uma questão importante na aplicação de uma teoria de aprendizagem é o sequenciamento da instrução. A ordem e organização das atividades afetam a maneira da informação ser processada e como ocorre a aprendizagem (Glynn \& Divesta, 1977; Lorch \& Lorch, 1985; Van Patten, Chao, \& Reigeluth, 1986). Existe pouca discussão em torno de uma metodologia genérica para o desenvolvimento do OA mostrando como construí-lo e dando suporte ao seu uso instrutivo. Daí a importância de uma teoria esboçar a maneira de projetar o sequenciamento da instrução. Partindo dessa premissa serão analisadas, neste artigo, as teorias de aprendizagem que evidenciam a organização 
da instrução mostrando que essa sequência visa contribuir para a melhoria da aprendizagem. Também serão apresentadas teorias instrutivas que orientaram a seqüência e o espaço de um OA, na concepção de Wiley (2000). E finalmente, será demonstrado como pode ser utilizado na prática no planejamento de quatro OAs, de acordo com as teorias analisadas.

\section{A sequência da instrução}

Já vem de muitas décadas que autores de teorias de aprendizagem vêm mostrando que a sequência da instrução é fundamental para que o aluno faça uma boa aprendizagem. Para que ela ocorra, as atividades precisam envolver o aluno, motivandoo na busca do significado de algum conceito ou idéia. As atividades devem estar estruturadas em torno de um tema, para que o aluno não desvie sua atenção, não se disperse em seus estudos.

Wertheimer (1923) enfatizou que o foco da teoria da Gestalt era a idéia de "agrupamento", ou seja, as características dos estímulos levam o estudante a estruturar ou a interpretar um problema de uma determinada maneira. Os principais fatores que determinam o agrupamento é proximidade, semelhança, encerramento e simplicidade. Pela teoria da Gestalt as instruções devem estar agrupadas pela proximidade ou semelhança entre os itens abordados, ou ainda, os itens são agrupados em conjunto se eles tendem a concluir com alguma similaridade. Em uma instrução, os itens serão organizados em simples números de acordo com a simetria, a regularidade e a igualdade.

$\mathrm{Na}$ teoria construtivista, Bruner (1966) afirma que a aprendizagem é um processo ativo em que os alunos constroem novas idéias ou conceitos baseados em seu conhecimento passado. Por esse motivo os currículos devem ser organizados em uma forma espiral de modo a que os alunos construam continuamente sobre o que eles já aprenderam. A instrução está relacionada a contextos e experiências pessoais.

Ainda segundo o mesmo autor, uma teoria da instrução deve abordar quatro aspectos principais: predisposição para a aprendizagem; a maneira em que o conhecimento pode ser estruturado de modo que possa ser facilmente entendido; a sequêencia mais eficaz para a apresentação do material; a natureza e o ritmo de recompensas e castigos (avaliação).

Ausubel (1968), afirma que a aprendizagem, no processo de ensino, necessita fazer algum sentido para o aluno. $\mathrm{O}$ autor entende que a aprendizagem significativa se verifica quando as informações no plano mental do aluno se revelam, através da aprendizagem por descoberta e por recepção. O processo utilizado para as crianças menores é o de formação de conceito, envolvendo generalizações de interesses específicos para que, na idade escolar já tenham desenvolvido um conjunto de conceitos, de modo a favorecer o desenvolvimento da aprendizagem significativa. Por isso, ele sugere para esse processo a utilização de organizadores prévios que são informações e recursos introdutórios, que devem ser apresentados antes dos conteúdos da matriz curricular, com a função de servir de ponte entre o que o aluno já sabe e o que ele deve saber. Os organizadores se tornarão mais eficazes se forem apresentados no início das tarefas de aprendizagem para que suas propriedades possam integrar-se como elemento atrativo para o aluno, visando provocar o interesse e desejo de aprender. Sua formulação deve contar com um vocabulário bastante familiar ao aluno, de modo que, sua organização, bem como a aprendizagem sejam consideradas como material de valor pedagógico.

Landa (1976) afirma que executar uma tarefa ou resolver um problema requer um sistema de conhecimentos elementares (unidades e operações). Existem classes de problemas que necessitam de uma sequência bem estruturada e predefinida, para executar as operações (problema algorítmico). Para este tipo de classes de problema, é 
possível formular um conjunto de instruções precisas (algoritmos), a fim de resolver quaisquer problemas que pertençam a esta classe. Também existem classes de problemas (problemas criativos ou heurísticos), que não têm conjuntos de instruções precisas. Para estas classes de problemas é possível formular instruções que contenham certo grau de incerteza (heurísticas).

Nessa teoria, Landa (1976) sugere que todas as atividades cognitivas podem ser analisadas em todas as operações de natureza algorítmica, semi-algorítmica, heurística ou semi-heurística. Uma vez descoberto, as operações e os seus sistemas podem servir como base para a instrução de estratégias e métodos. No que diz respeito à sequiência de instruções, ele propõe uma série de estratégias, sendo a mais importante o método da "bola de neve". Esse método se aplica ao ensino de um sistema de operações cognitivas através do ensino da primeira operação, em seguida, da segunda, que é praticado com a primeira, e assim por diante.

Scandura (1977) explicou que na teoria estrutural da aprendizagem, o que é aprendido são regras compostas de um domínio, alcance e procedimento. A estrutura da aprendizagem prescreve o caminho mais simples da solução para um problema complexo e, em seguida, ensina os caminhos até que toda a regra seja dominada.

Merril (1983) afirma que existem duas formas de apresentação de instrução: primárias e secundárias. Na Component Display Theory (CDT) ele especifica quatro formas primárias: regras, exemplos, generalização e práticas, já as formas secundárias de apresentação incluem: pré-requisitos, objetivos, ajudas, mnemônicas e feedback. Sendo que a instrução é mais eficaz na medida em que contém todas as formas primárias e secundárias. Assim, seguindo esse autor, uma instrução completa consiste numa seqüência de regras, exemplos, recordação, prática, feedback, ajuda e mnemônicas adequada à tarefa que está sendo aprendida.

Gagné (1987) sugeriu que a aprendizagem de tarefas para habilidades intelectuais pode ser organizada em uma hierarquia de acordo com a complexidade. $\mathrm{O}$ principal significado da hierarquia é identificar as condições prévias (pré-requisitos) que devem ser seguidos para facilitar a aprendizagem em cada nível. A hierarquia fornece uma base para o sequenciamento da instrução.

Pela proposta de Gagné, o desenvolvimento de estratégias instrucionais é a sequência de nove "eventos":

a) ganhar a atenção: o que pode ser obtido fazendo uma pergunta provocativa, ou apresentando um fato interessante, ou ainda, apresentando um problema de interesse imediato para o grupo;

b) descrever os objetivos: mostrando o que o aluno vai aprender e como ele vai poder utilizar o novo conhecimento;

c) estimular a conexão com o conhecimento anterior: pela explicitação da relação entre o novo e os conceitos já adquiridos;

d) apresentar o material a ser aprendido: na forma de gráficos, textos, simulações;

e) orientar a aprendizagem: através da apresentação de exemplos, estudos de caso, representações gráficas, material complementar;

f) propiciar desempenho: criando situações e oferecendo condições para a aplicação do novo conhecimento:

g) dar feedback: mostrando, imediatamente, o grau de acerto do aprendiz na aplicação do conhecimento;

h) avaliar: através de testes, o grau de assimilação do novo conhecimento.

i) aumentar a retenção e facilitar a transferência do conhecimento: através de exercícios de aplicação.

\section{Teorias instrutivas para a orientação do espaço e da seqüiência}


Um objetivo das teorias instrutivas de um OA é orientar a seqüência da instrução. Ao elaborar a Learning Object Design and Sequencing Theory (LODAS), Wiley (2000) fez a análise de quatro teorias instrutivas, as quais forneceram a identificação para a orientação do espaço e da seqüência dos OAs do seu projeto. Essas teorias estão sintetizadas abaixo, sendo mostrado as etapas da seqüência da instrução recomendada por esses teóricos.

\subsection{Teoria da Elaboração}

Reigeluth (1999) afirma que a teoria da elaboração ajuda os usuários "selecionar e arranjar os conteúdos em sequiência de uma forma a otimizar a realização de OAs". Nessa teoria, a instrução deve ser organizada em ordem crescente de complexidade. Por exemplo, para ensinar uma tarefa, primeiro é apresentada a versão mais simples; posteriormente serão adicionadas mais lições até que toda a tarefa seja ensinada. Em cada aula, o aluno deve ser lembrado de todas as versões vistas.

Reigeluth (1999) desenvolveu o Método da Simplificação de Condições (SCM), o qual fornece a informação relevante a respeito do espaço e da seqüência do conteúdo instrutivo. O SCM é composto de duas partes, sumário e desenvolvimento. Sumário significa encontrar a versão mais simples da tarefa que deve ser ensinada e que represente a tarefa inteira. Desenvolvimento significa ensinar aos estudantes versões cada vez mais complexas da tarefa. O SCM pode ser resumido nas seguintes etapas:

a) Preparar a análise do conteúdo e do projeto instrucional.

b) Identificar a versão mais simples da tarefa a ser ensinada, dando a atenção especial à simplificação das condições, isto é, as condições que tornam esta versão da tarefa mais simples do que a outra.

c) Analisar a organização do conteúdo para esta tarefa. A organização do conteúdo é realizada através de diferentes estratégias organizacionais pelos métodos processuais, heurísticos ou a combinação dos dois.

d) Analisar o conteúdo de apoio ou de pré-requisito.

e) Decidir o tamanho da instrução individual. Nem muito grande, nem muito pequeno, o tamanho apropriado é situacional e varia dependendo dos diferentes fatores como o tempo, a habilidade do aprendiz, a dificuldade do conteúdo, etc. As instruções não necessitam ser do mesmo tamanho.

f) Determinar a seqüência do conteúdo.

g) Identificar a versão seguinte da tarefa (primeira elaboração).

h) Analisar o conteúdo organizado, o conteúdo de apoio e determinar o tamanho e a seqüência do conteúdo (etapas c a e) para a versão seguinte da tarefa.

i) Retornar a etapa g identificando as versões restantes da tarefa e as instruções do projeto.

\subsection{Teoria da síntese do modelo de trabalho}

A síntese do modelo de trabalho fornece uma estrutura em que os objetivos individuais podem ser combinados em desempenhos significativos, reais, ou em modelos de trabalho (Bunderson at al., 1981, Gibbons at al., 1995; Gibbons e Fairweather, 1998).

Um modelo de trabalho não é um evento instrutivo. No entanto, é uma especificação em que diversos eventos instrutivos individuais podem ser criados. Esses autores afirmam que existem diversos tipos de objetivos para modelar o trabalho como descritos abaixo:

a) Muitos-para-um: em que muitos objetivos instrutivos são integrados em um único modelo de trabalho. Por exemplo, os objetivos "usar corretamente a pontuação" e "escrever corretamente o período das palavras" podem ser combinados num modelo de trabalho "escrever um ensaio". 
b) Um-para-muitos: onde um único objetivo instrutivo da tarefa modela trabalhos múltiplos. Por exemplo, os objetivos acima podem adicionalmente estar nos modelos de trabalho "criar um resumo" e "submeter um artigo para a publicação".

c) Um-para-um: onde um objetivo instrucional modela diretamente um único trabalho. Isso ocorre quando uma habilidade pré-requisito deve ser dominada antes que possa ser combinada em um modelo mais significativo do trabalho. Por exemplo, o objetivo "uso eficaz do software para processar a palavra" poderia ser praticado isolando outros modelos do trabalho listados acima.

\subsection{Teoria do domínio.}

A teoria do domínio (Bunderson, Newby, \& Wiley, 2000) pode ser vista como uma extensão da aproximação da teoria da síntese do modelo de trabalho aplicada à avaliação. Ela fornece a orientação do espaço e da seqüência atrelada à teoria fundamental. A teoria do domínio trabalha para representar o que as pessoas podem fazer com seu conhecimento e abstrair o que sabem.

Em sistemas instrucionais, o domínio é modelado em termos de conhecimento a ser aprendido. Isto é feito pela identificação da estrutura do conteúdo do domínio em termos de componentes tais como fatos, princípios, conceitos e procedimentos, e relações entre esses componentes, tais como dependências lógicas e hierarquias.

Essa teoria trabalha com o modelo de trabalho específico produzido pela síntese do modelo de trabalho e examina as construções subjacentes da técnica dentro do domínio dado. Por exemplo, na aquisição do domínio da linguagem, as construções subjacentes podem ser categorizadas nos grupos que escutam, falam, escrevem e lêem (Forte-Krause, 2000).

\subsection{Modelo do projeto instrucional dos quatro componentes}

$\mathrm{O}$ modelo do projeto instrucional dos quatro componentes (4C/ID) esboça uma maneira de projetar o treinamento que suporta a aprendizagem de habilidades cognitivas complexas (Van Merriënboer, 1997; Paas \& Van Merriënboer, 1992; Van Merriënboer \& Dijkstra, 1996; Van Merriënboer, Jelsma \& Paas, 1992). Esse modelo tem quatro etapas, descritas abaixo:

a) Princípio da decomposição da habilidade: nele a habilidade cognitiva complexa é quebrada e é treinada em um método recorrente (algorítmico) e em um método não-recorrente (heurístico);

b) Análise adicional destes dois métodos das habilidades constituintes que revelam o conhecimento que suporta estas habilidades;

c) Selecionar métodos instrutivos para praticar habilidades constituintes e apresentar a informação suportada (a ênfase está mais na prática ativa do que na apresentação da informação);

d) Compor uma estratégia do treinamento (Van Merriënboer, 1997).

Essa teoria é atrelada em uma quantidade significativa de pesquisa e fornece a sustentação específica nos termos das estratégias instrutivas ensinando os dois tipos de habilidades. As habilidades recorrentes, ou as habilidades que são executadas sempre da mesma maneira, são ensinadas usando a prática da tarefa e o conhecimento do prérequisito (Van Merriënboer, 1997; Van Merriënboer \& Dijkstra, 1996). Por exemplo, "adicionar três dígitos de números" é executada sempre da mesma maneira, sendo uma habilidade recorrente. Esta habilidade pode ser dividida em duas sub habilidades, "adicionar um dígito numérico" e "carregá-lo". A prática da tarefa recomenda que cada uma destas habilidades seja ensinada e praticada até que seja automatizada. Toda a informação que o aprendiz necessitar para executar estas tarefas está disponível durante a prática. 
As habilidades não-recorrentes, ou as habilidades que são executadas diferentemente em situações distintas, são ensinadas usando a prática da tarefa inteira e seu conhecimento é apresentado de maneira que promova a elaboração e a compreensão (Van Merriënboer, 1997; Van Merriënboer \& Dijkstra, 1996). Por exemplo, a programação do computador é classificada como uma habilidade não-recorrente. $\mathrm{O}$ conhecimento suportado para esta habilidade, tal como as estruturas diferentes dos laços, tipos de variáveis, etc., é ensinado antes de modo que o aprendiz a tenha disponível, na própria memória, durante a prática. A prática da tarefa inteira significa que esta habilidade é ensinada com uma aproximação holística tal como o modelo das condições simplificadas (SCM).

Van Merriënboer (1997) identifica três níveis do espaço, ou granularidade: conjuntos da habilidade, tipos de caso e problemas específicos.

Quando as habilidades são identificadas, podem ser criados os objetivos comportamentais para cada uma. Van Merriënboer (1997) preferiu o método da teoria da elaboração (Reigeluth, 1999) para reintegrar as habilidades antes de ensiná-las; entretanto, afirma que algumas tarefas são complexas em relação à otimização do escopo em um nível suficientemente simples. A tarefa inteira é formada por conjuntos construídos a partir de duas a cinco habilidades relacionadas e compreende o nível mais elevado da granularidade no modelo 4C/ID. Dentro de cada um desses conjuntos da habilidade é colocado um número de tipos de caso. Os tipos de caso são as categorias de problemas e os exemplos que o aprendiz experimenta durante a instrução, e representa o nível médio da granularidade. Finalmente, dentro de cada tipo de caso há diversos problemas específicos. Estes são os exemplos e os problemas reais com que o aprendiz interage durante a instrução, e representa o nível mais baixo da granularidade deste modelo.

Van Merriënboer (1997) identifica também três níveis de seqüência: macro, meso e micro. No nível macro, os conjuntos das habilidades são requisitados de acordo com uma seqüência de parte da tarefa. Estes conjuntos devem ser requisitados tais que as habilidades no primeiro conjunto sejam "pré-requisito" para o sucesso no segundo, e assim por diante. Os conjuntos das habilidades devem ser os escopos de modo que um único conjunto requeira não mais do que 200 horas para aprender. O primeiro conjunto deve ser pequeno o bastante para que os aprendizes comecem a prática simples, mas correta, da versão de toda a tarefa dentro dos primeiros dias. O conjunto final deve ser grande $\mathrm{o}$ bastante para confiar em todas as habilidades identificadas na análise preliminar. No nível meso, os tipos de caso são requisitados de acordo com uma seqüência de toda a tarefa. Finalmente, no nível micro, esse modelo apresenta as opções de seqüência para os problemas específicos, os quais devem ser grandes o bastante para fornecer exemplos ou prática de uma habilidade específica.

Para as tarefas complicadas, onde haja a interação do aprendiz com os exemplos e os problemas, eles são ensinados do padrão simples à maneira mais complexa, para evitar a sobrecarga cognitiva. Entretanto, para promover a transferência das habilidades às situações problema-solução, Van Merriënboer (1997) recomenda encontrar formatos de nível baixo do problema (por exemplo, trabalho fora da aula) e variar a seqüência do problema aleatoriamente.

\section{A prática no planejamento de $\mathrm{OA}$}

Nesta seção é mostrada a utilização prática das teorias analisadas, no planejamento de OA.

\section{1-Conceito de números primos seguindo a teoria do construtivismo:}


No enfoque da teoria construtivista, o conceito de números primos é facilmente entendido quando a criança constrói o quadro chamado Crivo de Erastóstenes, sugerido por Bruner (1973), percorrendo as seguintes etapas, elaboradas pela autora:

- escrever os números naturais em ordem crescente: $1,2,3,4,5 \ldots$

- eliminar, a partir do número 4, os múltiplos de 2 ;

- eliminar a partir do número 6, os múltiplos de 3;

- eliminar a partir do número 10, os múltiplos de 5,... e assim sucessivamente.

Faz-se a criança observar os números que não forem eliminados e somente após esse passo que esses números são chamados de primos. Fazer a criança notar que esses números só podem ser "eliminados" quando for múltiplos de 1 ou do próprio número estudado.

É fácil para a criança ir desta etapa para o reconhecimento de um número composto ou de um número primo, pois nessa construção a criança aprende a fatoração (eliminar, a partir do), os números compostos (múltiplos de) e os números primos.

\section{2-O raciocínio da operação de subtração seguindo a teoria estrutural da aprendizagem}

A teoria estrutural da aprendizagem de Scandura (1977) dá o suporte teórico para o raciocínio da operação de subtração, como é mostrado nas seguintes etapas do exemplo abaixo:

- selecionar uma amostra representativa da operação, em ordem crescente de complexidade. Por exemplo: 9-5, 248-13 e 801-302.

- identificar as regras de resolução de cada um dos problemas selecionados. Para realizar esta etapa, é necessário determinar as capacidades mínimas (prérequisitos) dos estudantes (por exemplo, reconhecer os dígitos 0-9, sinal, colunas e linhas). Em seguida, definir as capacidades mínimas do estudante, no exemplo, definir as modalidades das operações envolvidas na resolução de cada um dos problemas. A regra da subtração é o "empréstimo", este procedimento especifica se o maior número é inferior ao menor número de uma coluna (lembrando a idéia de unidade, dezena, centena,...), o número no topo da coluna para a direita deve ser feita por pequenos 1 (empréstimo).

- identificar todas as regras (mais ou menos abrangentes) para eliminar regras repetidas. No caso da subtração, pode-se substituir uma série de regras parciais com uma única regra para o empréstimo que abrange todos os casos.

- o último passo é testar e refinar o resultado da(s) regra(s) usando novos problemas e estendendo a regra estabelecida, se necessário, para que ela representa todos os problemas no domínio. No caso da subtração, podem-se ter problemas com a utilização de combinações de diferentes colunas (unidade, dezena, centena,...).

\section{3-O reconhecimento de um triângulo eqüilátero seguindo a teoria das condições de aprendizado:}

Seguindo a sequiência instrucional de Gagné (1987), o reconhecimento do triângulo eqüilátero é realizado através da seqüência de nove procedimentos:

- ganho da atenção - mostrar a variedade de triângulos gerados por computador;

- identificar o objetivo - colocar a pergunta: "O que é um triângulo eqüilátero?";

- pré-requisito - rever as definições de triângulos;

- estímulo - dar a definição do triângulo eqüilátero; 
- guia da aprendizagem - mostrar exemplos de como criar triângulos equiiláteros;

- obter desempenho - pedir aos alunos para criar cinco diferentes exemplos de triângulos equiláteros;

- fornecer feedback - verificar todos os exemplos criados pelo aluno como correto/ incorreto;

- avaliar o desempenho - fornecer os escores e a re-aprendizagem nos exemplos incorretos;

- melhorar a retenção/transferência - mostrar figuras de triângulos e pedir aos alunos para identificar triângulos eqüiláteros.

\section{4-Conceituar as leis da oferta e da demanda seguindo a teoria da elaboração:}

O aprendizado das leis da oferta e da demanda seguindo a teoria da elaboração de Reigeluth (1983) segue a seqüência:

- Preparar a análise do conteúdo das leis da oferta e da demanda, dentro da disciplina de Economia, mostrando que o modelo matemático dessas leis vem do modelo de funções matemáticas.

- Identificar a versão mais simples da tarefa a ser ensinada: enunciar as leis através de princípios.

- Organizar o conteúdo através do método heurístico - as leis da oferta e da demanda são enunciadas pelos princípios:

a) Um aumento no preço provoca um aumento da quantidade fornecida e uma diminuição na quantidade exigida.

b) Uma diminuição no preço provoca uma diminuição da quantidade fornecida e um aumento na quantidade exigida.

- Conteúdo de apoio: conceitos de preço, de oferta, de demanda, de aumento e de decréscimo. Conceito de funções lineares e quadráticas.

- Decidir o tamanho da instrução individual. Por exemplo, para cada princípio, trabalhar com dois exemplos práticos.

- Determinar a seqüência do conteúdo: objetivo; pré-requisito: modelo matemático de funções; conteúdo de apoio: conceito de preços, de oferta, de demanda, de aumento e de decréscimo. Enunciar os dois princípios. Analisar os exemplos de cada princípio.

- Identificar a versão seguinte da tarefa (primeira elaboração). Observar que praticamente todos os princípios da economia podem ser encarados como elaborações da lei de oferta e demanda, incluindo a fixação de preços, economias planificadas, etc.

- Analisar o conteúdo organizado, o conteúdo de apoio e determinar o tamanho e a seqüência do conteúdo para a versão seguinte da tarefa.

\section{5-Considerações finais}

Analisando as teorias anteriores conclui-se que, de uma maneira geral, a sequiência da instrução deve ser do simples para o complexo, sendo que a sequiência deve ser ditada pelo pré-requisito e o processo cognitivo envolvido.

De acordo com os autores pesquisados, os OAs devem ser apresentados em ordem crescente de complexidade, começando com o sumário ou o caso mais simples. A seqüência deve estar em uma ordem que simule o desempenho real com o nível crescente de dificuldade. 
Mas a seqüência também pode ser de acordo com seus níveis: macro (conjuntos de habilidades), meso (tipos de casos) e micro (problemas específicos). Os conjuntos das habilidades devem ser arranjados em seqüência em uma parte da tarefa, significando que as habilidades são ensinadas uma de cada vez e combinadas gradualmente. Os tipos de caso devem ser arranjados em seqüência de acordo com a ordem da tarefa, em que todas as habilidades são ensinadas simultaneamente. Os problemas específicos podem ser arranjados em sequiência na ordem "simples para complexa", ou quando praticáveis, em uma seqüência aleatória a fim de promover a transferência cognitiva.

Os OAs não necessitam ser todos do mesmo tamanho. Quando for pequeno pode ser combinado com outro objeto que o torne grande o bastante para ensinar desempenhos significativos. Mas acima de tudo, a sequência da instrução em um OA deve contribuir para que a informação seja processada pelo aprendiz de forma que este tenha uma aprendizagem significativa.

\section{Referência bibliográfica}

Ausubel, D.P. (1968). Educational Psychology: A Cognitive View. New York, Holt, Rinehart and Winston.

Bruner, J. (1966). Toward a Theory of Instruction. Cambridge, MA: Harvard University Press.

Bruner, J. (1973). Going Beyond the Information Given. New York: Norton.

Bunderson, C. V., Martinez, M., \& Wiley, D. (2000, April). Verification in a design experiment context: Validity argument as design process. Symposium session at the annual meeting of the American Educational Research Association, New Orleans, LA. Available: http://edumetrics.org/research/presentations/aera2000- wiley01.pdf

Bunderson, C.V., Gibbons, A.S., Olsen, J.B., and Kearsley, G.P. (1981). Work models: Beyond instructional objectives. Instructional Science, 10, 205-215.

Dahl, O. J. \& Nygaard, K. (1966). SIMULA - An algol based simulation language. Communications of the ACM, 9 (9), p. 671-678.

Gagné, R. (1987). Instructional Technology Foundations. Hillsdale, NJ: Lawrence Erlbaum Assoc.

Gibbons, A.S., Bunderson, C.V., Olsen, J.B., and Rogers, J. (1995). Work models: Still beyond instructional objectives. Machine-Mediated Learning, 5(3\&4), 221-236.

Gibbons, A. S. \& Fairweather, P. G. (1998). Computer-based instruction: design and development. Englewood Cliffs, NJ: Educational Technology Publications.

Glynn, S.M. \& DiVesta, F.J. (1977). Outline and hierarchical organization for study and retrieval. Journal of Educational Psychology, 69(1), 69-95.

Landa, L. (1976). Instructional regulamento e Controle: Cibernética, Algorithmization, metaheurísticas e na Educação. Englewood Cliffs, NJ: Educational Technology Publications.

Lorch, R.F. Jr., \& Lorch, E.P. (1985). Topic structure representation and text recall. Journal of Educational Psychology, 77(2), 137-148.

Merrill, M.D. (1983). Component Display Theory. Em C. Reigeluth (ed.), Instructional Design teorias e modelos. Hillsdale, NJ: Erlbaum Associates. Paas, F.G.W.C., \& Van Merriënboer, J. J. G. (1992). An instructional design model for the training of complex cognitive skills. Tijdschrift voor Onderwijsresearch, 17, 1727.

Reigeluth, C. \& Stein, F. (1983). The elaboration theory of instruction. In C. Reigeluth (ed.), Instructional Design Theories and Models. Hillsdale, NJ: Erlbaum Associates.

Reigeluth, C. M. (1999). The elaboration theory: Guidance for scope and sequence decisions. In C. M. Reigeluth (Ed.), Instructional design theories and models: A new 
paradigm of instructional theory. (pp. 5-29). Hillsdale, NJ: Lawrence Erlbaum Associates.

Scandura, J.M. (1977). Problem Solving: A Structural/Process Approach with Instructional Applications. NY: Academic Press.

Van Merriënboer, J. J. G. ; Jelsma, O.; Paas, F.G.W.C. (1992.) Training for reflective expertise: A four-component instructional design model for complex cognitive skills. Educational Technology Research and Development, 40(2), pp. 23-43.

Van Merriënboer, J. J. G., \& Dijkstra, S. (1996). The four-component design instructional design model for training complex cognitive skills. In R.D. Tennyson, F. Schott, N. Seel, and S, Dijkstra, (Eds), Instructional design: international perspectives. Mahwah, NJ: Lawrence Erlbaum Associates.

Van Merriënboer, J. J. G., (1997). Training complex cognitive skills: A fourcomponent instructional design model for technical training. Englewood Cliffs, NJ: Educational Technology Publications.

Van Patten, J., Chao, C.I. \& Reigeluth, C.M. (1986). A review of strategies for sequencing and synthesizing instruction. Review of Educational Research, 56(4), 437-471.

Wiley, D. A. Learning object design and sequencing theory. Doctoral dissertation, Brigham Young University. 2000.

Wertheimer, M. (1923). As leis de organização perceptual em formulários. Publicados pela primeira vez como Untersuchungen zur Lehre von der Gestalt II, em Psycologische Forschung, 4, 301-350. Tradução publicada em Ellis, W. (1938). Uma fonte livro de Gestalt psychology (pp. 71-88). London: Routledge \& Kegan Paul. [disponível em http://psy.ed.asu.edu/ clássicos / Wertheimer / Formulários / forms.htm] 